\title{
Implant survival in augmented maxillary sinus is more variable than that of implants placed in posterior maxilla
}

\author{
Is there any difference in implant survival and complications between \\ implants placed in augmented maxillary sinus and non-augmented posterior \\ maxilla?
}

\author{
Graziani F, Donos N, Needleman I, Gabriele M, Tonetti M. \\ Comparison of implant survival following sinus floor \\ augmentation procedures with implants placed in pristine \\ posterior maxillary bone: a systematic review. Clin Oral Implants \\ Res 15, 2004; 677-682
}

Data sources Cochrane Central Register of Controlled Trials, Medline and Embase were searched to April 2004. Key journals were hand searched to April 2004, together with bibliographies of all retrieved papers and review articles.

Study selection For inclusion, an article had to satisfy the following criteria: studies had to be controlled trials comparing implant placement in the non-augmented posterior maxilla with the augmented maxillary areas; study groups with at least 20 patients; number of implants and duration of follow-up were reported. The outcome measures were implant survival, changes in radiographic peri-implant bone height and complications. Unloaded implants were excluded.

Data extraction and synthesis The methodological quality of included studies was assessed evaluating blinding of the examiners and completeness of follow-up. All quality assessments were conducted in duplicate and independently. Data were synthesised in evidence tables and range of survival data and types of complications were summarised. Data were stratified for patients or implant-based analysis. Decisions on possible meta-analysis were made on the similarity between the studies. Results The search identified 579 articles, of which six (five studies) met the inclusion criteria. Implant survival ranged from 73 to $100 \%$ for nonaugmented sinuses and from 36 to $100 \%$ for augmented sinuses in patient-based data. From implant-based data, survival varied between 75 and $100 \%$ for both nonaugmented and augmented areas. Heterogeneity of the studies prevented meta-analysis.

Conclusions Implant survival appears to show greater variability in grafted sinuses than in the posterior maxilla. However, well-designed prospective studies with larger patient numbers and control of confounding factors are urgently needed.

\section{Commentary}

Once more, a systematic review of a dental operative intervention has exposed that the science basis for the practice is very weak. This time it is a surgical procedure, where the outcome of the intervention, at least in theory, should be open for assessment after relatively short observation periods, at least for many of the relevant clinical and patient-centred outcomes. Apart from experiencing that bone can be formed when there is none by a sinus floor augmentation procedure, we cannot provide unbiased advice to our patients about the merits of the procedures. We simply do not know whether the best results are obtained with, for example, an iliac bone block, an iliac + mandibular bone block, an iliac bone block + cancellous chips, an iliac bone block + Hydroxyapatite (HA), HA alone, demineralised freeze-dried bone allograft (DFDBA) alone, DFDBA and HA, an intraoral bone block + HA, Tricalcium phosphate (TCP), bovine porous bone mineral (BPBM) alone, $\mathrm{BPBM}+$ symphysis bone, collagen-sheet or -sponge, etc.

It is a strong leap of faith from our patients in general, when we can get away with this situation. How can we declare that patients consent is informed when we really can not provide an unbiased information to the patient in the first place? In another dimension is the phenomenon that many of the reports published in dental journals do not stand up to a critical appraisal. Why? It should not be a reflection of a laissez-faire attitude of a profession, where the meticulous attention to details is a core element of its existence. It cannot be too stringent critical appraisal criteria, since these are being applied to almost all spheres of biomedicine. It must rather be the low thresholds required for getting something published in any of the 700 dental journals that are available today. I spend far too much time browsing reports that fail to clarify sound internal and external scientific validity, although usually full of details of the clinical procedures. Sometimes one wonders whether it is the study itself that is inadequate or simply inadequate writing.

\section{Practice point}

- If I should give some advice I would suggest first, never carry out single-centre studies - the chances are that your study will be underpowered and prone to type 2 errors and second, always report your findings according to guidelines for trials reporting, of which the most important is the CONSORT format (www.consortstatement.org/).

\section{Asbjørn Jokstad}

Institute of Clinical Dentistry, University of Oslo, Norway

Evidence-Based Dentistry (2005) 6, 99.

doi:10.1038/sj.ebd.6400343
Address for correspondence: Filippo Graziani, Eastman Dental Institute, Department of Periodontology, 256 Gray's Inn Road, London WC1X 8LD, UK. E-mail: f.graziani@ eastman.ucl.ac.uk 